uimbus und strato-cumulus gehören bekanntlich $\mathrm{zu}$ len tiefststehenden Wolkenformen. Man kann auch sehr deutlich beobachten, daß die Olflecken sich um so deutlicher an den gekräuselten Teilen der Seeoberflache abheben, je energischer die Wolkenbildung ist und je tiefer an Horizont sie auftritt.

Die den Wolken ausgesetzte Wasserschicht iot kühler als ihre Umgebung, sie winkt etwas unter, wïrmere Schichten aus der Umgebung strömen nach, anch diese werden unter dem Einflub der Wolken stärker abgekuhlt, als anderswo und so bildhet sich schnell eine Stelle im Oberflächenwasser, die spezifisch schoerer als ihre Umgetung ist und dadurch der durch leichten Wind bewirkten Wellenbewegung der Oberfluchenschicht Widerstand bereitet. Es entsteh also an dieser Stelle für die Wasserbewegung ein Knotenpunkt, besser gesigt eine Knotenfläclse, von der :us die Wellen wieder zurückkehren. Sind die Ur wachen der ungleichmäBigen Erwirmung verseliwunden, l. h. haben die Wolken ihren Standpunkt, ihre Größe und Dichte geändert, 60 ist damit auch die Erscheinung der olflecke verschwunden bzw. haben sich ihr Standpunkt und A.usdehnung veründert und somit erklärt sich m. E. das blitzschnelle Auftreten und Wiederverschwindien, sowie die so äuBerst wechselnde und komplizierte Form der olflecken als eine durch ther mische Zustandsänderungen verursachte Intcrferenzerschcinung an Wasserwellen.

Allerdings ist as mir bisher nicht gelungen, Temperäturunterschiede $z$ wischen taches d'huile unxl anderen Sreteilen zu bonstatieren, da mir kein Instrument zur Ferfügung stand, die Temperatur so dinner Flïssigkeitsschichten, wie sie hier auftraten, messen 71 können. Um so mehr war ich erstaunt, daB $G$ schneider einmal in nur $4 \mathrm{~m}$ tielem Oberse be Reval am 30. Juli 1904 die Oberflichentemperatur riner glatten Stelle um $1,3^{\circ}$ kühler fand, als die jenige einer gekräuselten in ihrer unmittelbar'n Nühe. eive Beobachtung, deren wissenschaftlicher Wert mir sehr zweifelhaft vorkommt, weil Sohneider kein anderes Instrument zur Verfügung stand, als das bekannte Quellenthermometer. (Archiv für Biontologie, Tleranseg. von der Ges. naturw. Freunde in Berlin, Bd. II, Berlin 1908.)

Jedenfalls wird es einer verfeinerten Terhuik in der Erforschung der Thermik eines Sees noch gelingen, div Temperatur auch sehr dünner Flüssigkeitsschichten fest astellen, und dann wird eine experimentelle Bestïtigung meiner Theorie m. E. nicht ausbleiben. DaB dive Ausbreitung öliger Bestandteile iiber einzelne Teile des Sees selbstverständlich auch häufig zur Bildung der taches d'huile beitragen kann, betonte ich bereits oben. Zum SchluB möchte ich noch darauf hinweisen. dub es neben ,objektiven" Olflecken auch ,subjektive" gibt, die vom Standpunkt des Beobachitens abhängen; der Unterschied beider Erscheinungen lïßt sich leicht feststellen, wenn mehrere Beobachter gleichzeitig an verschiedienen Stellen aus einer bestimmten Gegend des Sees beobachten, wobei besonders darauf zu achten ist. daß die vertikale Entfernung der Beobachtang vom See dabei eine entscheidende Rolle spielt. Das Kapitel der Olflecken bietet ein dankbares Feld für die Beobachtungskunst der Anvolner eines Sees und verliente eine eingehende monographische Bearbeitung.

Jena, den 1. Juli 1917.

Prof. Dr. W. Halbiaß.
Kulturverfahren zur Vermehrung der Getreideerzeugung.

In Nr. 23 der "Naturwisenschaften" d. J. findet sich Seite 387 ein Referat aus den Compt. rend. 164, 1917, in dem uber ein Kulturverfahren Devanis zur Vermehrung der Getreideerzengung berichtet wird.

Hierzu habe ich folgendes zu bemerken:

Zur Anzucht krïitiger, reichblühender Pflanzeu werden in unserem bota nischen Garten die Samen einjährig äberwinternder Pflanzen: Digitalis purpurea, Campanula Medium, Oenothera Lamarckiana, Viola tricolor muxima u. a, bereits Ende Juli in die Früh. beete ausgesät, die jungen Pflanzen alsdann vertopft und anfungs September ins freie Land gesetzt. Bis zum Schlub der Vegetationsperiode entwickeln sie sich so weit, dab sie schmeelose Winter unter einer leichten Fichtenreisigdecke gut iiberdauern.

Diese gärtnerische Erlahnung wandte ich anfangs Auguet 1915 auf Roggen (Schlanstedter) an. Die ausgesüten Getreidekörner entwickelten noch in demselbeu Jahre Büschel bis zu $20 \mathrm{~cm}$ Durchmesser. Der Boden war nicht gediingt, hielt sich aber infolge seiner geschützten Lage und der großen Absorptionsfähigkeit des Auenlehms für Wilsser gleichmäBig feucht. Am 23. Mai 1916 blühte das ganze 10 qm große Roggenbeet. Aus jedem Büischel waren $6-8$ Halme aufgeschossen. Die Halmhöhe betrug $160 \mathrm{~cm}$ im Durch. schnitt. Ein Behäufeln und Verpflanzen der Getreinlebüschel hatte nicht stattgefunden, dawit ein Vergleich mit der landwirtschaftlichen Kultur leichter durchführbar blieb. Das Ernteergebnis übertraf dass eines Parallelversuches mit Wintergetreide derselbelı Rasse in seiner Körnermenge beinahe um das Doppelte, in seiner Strolugewichtsmenge um das Dreifache. Der Roggen war 14 Tage irüher roif gegenüber dem in Oktober zur Erde gebrachten Saatgnite.

Der Versuch latte zunächst orimtierenden sinn. Ob deshalb die in leihe aufgewachsen'n Roggenbüschel je einem Samenkorn ilıren Ursprung verdanken oder ilts einem Komplex ron Finzelpflanzen bestanden. wurde nicht ermittelt. Nach dieser Riclitung soll in diesem Jahre die Kultur geleitet werden; ebenso muls, wenn die Rentabilitiit des Verfahrens geprifit werden soll, das Verhältnis der Aussaatmenge zum Erntegrewichtsergebnis zahlenmäBig festgelegt werden.

Die meteorologischen Elemente: Boden- und Luittemperatur, Feuchtigkeitsgehalt u, a. wiihrend der Zeit vom August 1915 bis Juni 1916 wurden dagegen taig. lich gemessen.

Die klimatischen Faktoren sind ansschlaggebend bei diesem fruhzeitigen Kulturverfahren. Zweifellos versprechen nur ganz günstig gelegene örtlichkeiten $\mathrm{Er}$ folg. Der Grobbetrieb muB jedoch so eingestellt sein, daB er unter allen Lmständen mit Ernteertirag rechnen kann. Diesen gewaibrleistet die ïbliche Oktobereinsaat.

Leipzig, den 24. Juni 1917.

$$
\text { Dr. B. Stange. }
$$

\section{Berichtigung zu dem Aufsatze: Der Streit um das Elektron.}

Ich entspreche einem Wunsche des Herrn Elirenhafl, indem ich folgende Bemerkung nachtrage. Ich hatte $e s$ in meinem Aufsatze als zweifelhaft hingestellt, ob Herr Ehrenhaft das Prinzip der Messung der Einzelladungen selbständig gefunden hat, oder ob er durch Millikans Mitteilung darauf lingeführt worden ist. Mir war dabei eine ganz kurze Notiz entgangen, die IJerr Ehemhaft schon im März 1909 im Wiener An. 
zeiger veröffentlicht hat. In dieser hat er schon damals die Möglichkeit der Messung der Einzelladungen richtig erkannt und im Prinzip beschrieben. Ich wheche dies hiermit ausdrücklich anzuerkennen und meine Darstellung in diesem Punkte zu berichtigen.

Gießen, den 29. Juni 1917.

$$
\text { Prof. Dr. Walter König. }
$$

\section{Gesellschaft für Erdkunde zu Berlin}

In der Fachsitzung am 21. Mai hielt Dr. R. Prietze (Berlin) einen Vortrag über ein Vermelichtnis von Barth und Nachtigal, in dem er zunächst einen Uberblick uber die Erforschung des Sudans und seiner Volksstämme gab und sodann die Verdienste der beideı Heroen aus jener klassischen Epoche der deutschen Afrikaforschung eingehend würdigte. Der erste Vertreter der Mittelmeerkultur, der den König von Bornu besuchte, war Leo Africanus, ein getaufter Maure, der zu Beginn des 16. Jahrhunderts ausgedehnte Reisen in Nordafrika unternommen hatte.

Der Schwerpunkt der Völkerkunde Zentralafrikis liegt in der Kenntnis der Sprachen, und darum ist die linguistisclie Forschung, die im wesentlichen eine Frucht dentschen Schaffens ist, als ethnologische Leistung ersten Ranges zu bewerten. Trotz ihrer großen Er. folge auf geographischem wie ethnographischem Gebiet haben weder Barths sechsjuhrige, noch Nachtigals fünfjithrige Reisen den Kostenaufwand von 10000 Talern trreicht. Trotzdem sie unter den wilden, häufig von religiösem Fanatismus ergriffeneu Stämmen nie ihr Clıristentum verleugneten und häufig in Lebensgefahr wchwebten, haben sie niemals auf afrikanischem Boden ein Gewehr abgefeuert. Eingehend schilderte der Vortragende, wie mühevoll und zeitraubend die granmatiknlisehe Forschung in solchen Ländern ist, wie die Neger die Frage nach dem Namen eines Flusses oft nur mit dem Wort „Wasser“, nach demjenigen einer Pflanze mit dem Wort "Kraut" beantworten. Alle diese Schwierigkeiten wubten beide Forscher mit Geschick zu bemeistern. Die Kenntnis von Land und Volk ist uns erst erochlassen, wenn die Sprache der Bewohner erforscht ist, und ohne diesen Ariadnefaden ist es nicht möglich in das Labyrinth der geistigen Fultur und der historischen tberlieferungen der ein. zeluen Stimme einzudringen.

Der Hanburger Heinrich Barth machte Bornu, siillich des Tschadsee, zu seinem Operationszentrum und erforschte von dort auf ausgedehnten Reisen, die sich zusammen auf rund $25000 \mathrm{~km}$ erstrecken, die Stantengebilde des Sudans, wobei sich eine kulturelle cberlegenheit des Westens uber den Osten feststellen lieB. Barth hielt sich als erster Europaier liingere Zeit in Timbuktu auf, dem afrikanischen Athen, das frither eine Bibliothek von 1600 Bänden beherbergt liatte und ein Zentrum afrikanischer Gelehrsamkeit gewesen war. Eine besonders große Anziehungskraft übten die beiden großen Völkerfamilien der Fulbe und der Haussa auf Barth aus. Der Durchschnittsbetrag der von ihm für jede Sprache gesammelten Worter iibersteigt 2000. Leider aber fand er keine $\mathrm{MuBe}$, sich mit Erzăhlungen aus dem Munde der Eingeborenen zu beschäftigen, die doch die wahre Quintessenz linguistischer Studien sind. Auch ein Mangel an plastischer Gestaltungskraft haftete ihm an, und sein Werk blieb unvollendet.

Gustav Nachtigal aus Eichstedt bei Stendal, der naturwissenschaftlich gebildete Arzt, erginzte in glück. lichur Weise die Arbeit des Historikers. Er war als einziger Europiler nach Tibesti gekommen, jenem höchsten Gebirgslande in der ostlichen Sahara, wo er mit knapper Not dem Tode entrann. Er erforschte unter Einsetzung soines Lebens Wadai in Osten des Tschadsee, nachdem er in Bornu das Kanuri, die am höchsten entwickelte und schönste der Sudanspracheu studiert batte. Die weitere Ausarbeitung seiner linguistischen Arbeiten sollte der Vortragende, der Sohn seiner Schwester, obernehmen, oin Plan, den anfangs ler Tod Nachtigals, später langjahrige Krankheit des Vortragenden vereitelte. Nach jahrelanger Vorbereitung durch Sprachstudien in verschiedenen Ländern Nordafrikas, Gewinnung zusammenhängender Texte, namentlich ron Liedern und Sprichwörtern, ist er jetzt in den Stand gesetzt, mit Unterstlitzung der Gesellschaft für Erdkunde die Weiterbearbeitung des im Besitze der Königlichen Bibliothek befindlichen liandechriftlichen Materials fortzusetzen: $O . B$.

\section{Mitteilungen aus verschiedenen Gebieten.}

Uber den Verlaul der alkoholischen Gärung bel alkallscher Reaktion. I. Zellfreie Gärung in alkalischen Lösungen; von Carl Neuberg und Eduard Färber. Bioch. Zeitschr. 78,238 . 1916. Nachdem Neuberg und Mitarbeiter irüher festgestellt hatten, daß einerseits im Reagenzglase Traubenzucker und seine Isomeren nicht nur durch Xtzlaugen, sondern auch durch schwach alkalisch reagierende Stoffe, wie Karbonate, Bikarbonate, Sulfite, Phosphate und Borate leicht in Methylglyoxal übergeführt werden können, und anderseits \%ymase gegen $0,5 n$-KOH viel weniger empfindlich ist, als man früher geglaubt hatte, konnte der Versuch gewagt werden, alkoholische Gärung bei alkalischer Reaktion vorzunehmen. Obgleich es als ausgemacht galt, daß die Gärung durch Hefe nur bei schwach saurer leaktion vor sich geht, zeigte es sich jetzt; daB sie auch in alkalischer Lösung durchgeführt werden kann. Geeignete Alkalisatoren sind die zuvor erwähnten schwach alkalisch reagierenden Stoffe. In ihrer Gegen. wart gären nicht nur Hexosen, sondern auch Rohrzucker; d. h. Invertase ist entgegen den bisherigen Voraussetzungen auch bei erheblicher OH-IonenKonzentration wirksam. Um ein klares Bild von diesen neuartigen Verhältnissen zu gewinnen und Beeinflussung der lebenden Substanz auszuschlieBen, kann man mit Hefensaft arbeiten. Solche rein enzymatischen Gäransatze vergären bei einem Zuckergehalt von $5 \%$ und Zugabe von $1 / 10$ Volumen an wiissriger Alkalisatorlösung in Gegenwart ron $10 \%$ Toluol (!) vollständig bei einer Gesamtalkalinität von 0,1 bis $0,2 \mathrm{~m}$-Dialkalikarbonat, Metaborat und Trialkaliphosphat, während die Konzentration an sekundärem Alkalisulfit nur $0,02 \mathrm{~m}$ entsprechen darf. Weiterhin ergab sich die grundsatzlich wichtige Tatsache, daB der Zeitpunkt des Alkalisatorzusatzes von wesentlicher Bedeutung ist. Fügt man nămlich die Alkalisatoren erst hinzu, nachdem das Saft-Zucker-Gemioch gerade zu gären begonnen hat, dann sind 2,5mal so hohe Alkalikonzentrationen anwendbar. Auch dabei verschwindet der Zucker vollständig.

Die Wirkung der alkalischen Zusilze auBert sich - und damit erlangt die ganze Erscheinung erhohte Bedeutung far die Erklärung des Zuckerabbaus bei der Garrang - in einer spezifiachen Veränderung der Garprodukte. Diese besteht in einer verminderten 